\title{
La clave cultural del Directorio para la catequesis
}

Salvatore Currò̀

\section{Resumen}

Este artículo intenta presentar cómo el Directorio para la catequesis (DC) aborda los desafíos culturales que plantea la situación actual a la evangelización y la catequesis. Para ello se sitúa en un primer apartado el DC en el marco de los últimos acontecimientos eclesiales que han marcado su renovación, así como el contexto cultural que ha motivado su cambio. En este sentido el artículo pone de manifiesto que el DC no ha podido recoger el desafío de la pandemia. Posteriormente se analiza cómo el DC afronta los desafíos de la relación entre la evangelización, la Revelación y la cultura en el marco de la transmisión de la Revelación, retomando los interrogantes de Evangelii Nuntiandi. Se realiza a continuación una presentación de los aspectos claves del DC en relación a la transmisión de la Revelación. El tercer apartado analiza cómo la clave cultural que está relacionada con la comprensión de la Revelación.

\section{Palabras clave:}

Directorio, evangelización, cultura, revelación, transmisión de la fe

\section{Introducción}

El contexto cultural actual desafía radicalmente a la Iglesia, a su evangelización y a su catequesis. ¿De qué manera el Directorio para la catequesis (DC) se enfrenta con el desafío cultural? ¿Cuál es la actitud prevalente en ella con relación a la cultura? ¿Hasta dónde incide la cultura en la comprensión misma de la catequesis y de la evangelización? La perspectiva cultural es la que hace resaltar las

1 Josefino de Murialdo, es profesor de la Universidad Pontificia Lateranense, de la Universidad Pontificia Salesiana de Roma. 
novedades del DC pero es también la que hace surgir su principal dificultad. La dificultad no es solamente del DC sino también de la pastoral catequística de las últimas décadas, que el DC recoge y vuelve a poner de actualidad.

La clave cultural será estudiada con una preocupación de contexto o de horizonte: ante todo será necesario situar el DC en el contexto del camino eclesial reciente para captar la razón cultural que ha exigido su publicación y que enseguida descubriremos en conexión con una razón teológica (1); nos detendremos, luego, en el capítulo I del DC, que describe el horizonte de comprensión de la catequesis y que establece una relación entre evangelización, Revelación y cultura, dentro de la perspectiva de la transmisión de la Revelación (2); finalmente, mostraremos en qué modo la clave cultural remite al núcleo (teológico) de la comprensión de la Revelación (3).

\section{1. ¿Con retraso sobre la cultura ya desde la salida?}

El DC fue publicado mientras el mundo entero se enfrentaba a la pandemia ${ }^{2}$ que, como ha señalado el Papa Francisco, ha destapado las falsas seguridades de la humanidad y nos provoca a construir un mundo renovado bajo el signo de la recíproca pertenencia como hermanas y hermanos ${ }^{3}$. La provocación cultural es fuerte y sería grave desperdiciar la llamada al cambio ${ }^{4}$. Evidentemente, la pandemia ha tenido también un gran impacto en la comunidad eclesial, ha provocado cambios o, mejor, ha puesto en evidencia posteriormente la exigencia de conversión (espiritual, pastoral, misionera) que desde hace tiempo se hace presente en el contexto social. Se han produci-

2 El DC fue aprobado por el Papa Francisco el 23 de marzo de 2020 y fue presentado en la Sala de Prensa del Vaticano el 25 de junio de 2020, con intervenciones de R. Fisichella, J. O. Ruiz Arenas, F.-P. Tebartz-Van Elst (las presentaciones pueden encontrarse en el sitio web del Consejo Pontificio para la promoción de la nueva evangelización: www.pcpne.va).

3 Papa Francisco, Meditazione in occasione del momento straordinario di preghiera in tempo di epidemia, Roma, Sagrato de la Basílica de San Pedro, 27 de marzo 2020; v. también Papa Francisco, Fratelli tutti, Lettera enciclica sulla fraternità e l"amicizia sociale, 3 de octubre 2020, 32-36.

4 El Papa Francisco lo ha expresado de manera eficaz: "Peor que esta crisis será sólo el drama de desaprovecharla" (Homilía en la solemnidad de Pentecostés, 31 de mayo 2020). 
do intentos de conversión pastoral: nuevas formas de solidaridad y de testimonio, experimentos litúrgicos, nuevos modos de entrar en contacto y de formar comunidad. Ha habido (y hay) muchos intentos incluso en el ámbito de la pastoral catequística; se ha buscado hacerse presente con mayor convicción en el mundo virtual y en el social; de pensar la pastoral y la misma comunidad más a partir de los individuos, de los pequeños grupos y de las familias, de experimentar nuevas formas de anuncio y de catequesis, más vinculadas a los desafíos de lo cotidiano y más a partir de signos de solidaridad y de intercambio. No está claro hacia dónde nos dirigimos, en el mundo en general, y también en la Iglesia y en la pastoral. Nos vamos moviendo, obligados a observar el futuro, provocados a arreglar cuentas con la provocación cultural y a construir algo nuevo.

En este contexto de éxodo, de desestabilización de los esquemas mentales, de búsqueda de nuevos paradigmas (culturales, sociales, económicos, culturales), el DC, en el momento de su publicación, ha podido aparecer para algunos casi ya superado por las circunstancias. De hecho, el mismo, no pudo tener en cuenta la pandemia. Pero si bien es cierto que la pandemia, aun siendo un viento nuevo e inesperado, ha puesto de manifiesto una crisis cultural (y eclesial) que ya estaba presente, la verdadera cuestión es si el DC está a la altura de semejante desafío, si nos ayuda a estar presentes en el mundo justamente en nuestro tiempo, si nos ofrece un paradigma pastoral-catequístico o criterios a la altura de los desafios de nuestro tiempo, y en consecuencia también de la pandemia. Los caminos más concretos, medidos con relación a problemas y contextos específicos, deberán encontrarse a nivel local ${ }^{5}$. Pero tales caminos, ¿podrán apoyarse en el DC? El horizonte cultural del DC. ¿se halla a la altura del cambio epocal que estamos viviendo? ¿Con qué actitud el DC enfrenta los desafíos culturales actuales? ¿Ayuda a ubicarlos e interpretarlos a la luz del Evangelio?

La interrogación sobre el núcleo cultural, que nos planteamos, intercepta, en realidad, la razón principal de la publicación del nuevo Directorio. El mismo, que además se sitúa en continuidad con los dos Directorios precedentes (1971 y 1997) quiere medirse a nuevos

5 El DC reconoce a las Iglesias particulares la tarea de la inculturación de la fe (394). 
fenómenos culturales. ¿Cuáles? La presentación pone en evidencia dos; interconectados entre ellos y que implican cambios radicales en la existencia de las personas: la cultura digital y la globalización de la cultura ${ }^{6}$. No obstante, la razón cultural debe ser comprendida en relación con el contexto eclesial y con las sensibilidades sobre ello que han ido madurando en los años recientes. Entre los eventos eclesiales, por su estrecha conexión con la catequesis, al menos, es necesario recordar: el sínodo sobre La nueva evangelización para la transmisión de la fe cristiana (2012); la constitución, después de ese sínodo, del Consejo Pontificio para la Promoción de la Nueva Evangelización, que será el nuevo encargado de la catequesis; la preocupación, difundida en algunos ámbitos eclesiales, de tener más en cuenta, de cuanto lo había hecho el Directorio General para la Catequesis (DGC), el Catecismo de la Iglesia Católica; la publicación de la Evangelii Gaudium (24 de noviembre de 2013) y los demás textos del magisterio del Papa Francisco; los sínodos más recientes (sobre todo aquellos sobre la familia y sobre los jóvenes). Tales eventos ${ }^{7}$ han marcado el proceso de preparación del nuevo Directorio ${ }^{8}$. Los mismos reflejan sensibilidades diversas sobre la evangelización y sobre el modo de entender la relación con la cultura; pero, todos ellos han reforzado, en algún modo, la idea de pensar sobre la catequesis en estrecha conexión con el proceso de evangelización.

La exigencia de hacer frente al desafío cultural actual está, en realidad, vinculada a la preocupación -teológica o teológico-pastoral - de reafirmar el primado de la evangelización y una comprensión de la catequesis a la luz de dicho primado. Esta razón "de tipo teológico", que se suma a la cultural está subordinada a la teológica; más exactamente: el desafío cultural debe encararse a partir de la

6 La Presentazione al Directorio ofrecida por S. Fisichella y J. O. Ruiz Arenas y también R. Fisichella, Guida alla lettura, en Pontificio Consiglio per la Promozione della Nuova Evangelizzazione, Direttorio per la catechesi, San Paolo, 2020, 7-8.

7 Recordado en Fisichella, Guida alla lettura, 9.

8 El proceso resultó laborioso: duró 6 años y pasó por 12 borradores; v. la intervención de J. O. Ruiz Arenas en la conferencia de prensa de presentación del Directorio, 25 de junio 2020; Cf. también R. Fisichella, "Le motivazioni di fondo, i punti neuralgici e la parole-chiave nella tessitura del nuovo Direttorio per la catechesi (2020)", en Salesianum 82/4 (2020) 616.

9 Como la llama Fisichella, Guida alla lettura, 10. 
preocupación de tal primado. Algo importante se ha decidido ya desde su partida. De ese modo, el DC confirma, aunque con algunas matizaciones, la comprensión de la catequesis que ya proponía el DGC. Lo cual, en verdad, no es poco si se piensa que, en los años concomitantes con el inicio del proceso de preparación del DC, se iba abriendo camino una tendencia que cuestionaba la renovación posconciliar de la catequesis, en nombre de una instancia "veritativa" que debía imponerse sobre la metodológica y comunicativa y que se apoyaba sobre el Catecismo de la Iglesia Católica y sobre su Compendio. La Evangelii Gaudium ha tenido un papel importante para limitar estas tendencias y en reafirmar el magisterio posconciliar sobre la catequesis ${ }^{10}$. Además, ha acentuado la comprensión kerigmática de la catequesis y su conexión con la evangelización"; evidentemente con la evangelización según es entendida en la Evangelii Gaudium.

Queda abierta la cuestión de si la idea de evangelización del DC es la misma que la de la Evangelii Gaudium. Alguna diversidad de matices existe, y a ello nos referiremos. Pero lo que se quiere tener en cuenta, e incluso problematizar, es si la relación primera de la catequesis debe ser con la evangelización; o mejor aún: si el horizonte de la evangelización debe ser el primero (o el último, en el sentido de lo fundamental) de la catequesis.

La hipótesis que me guía es que debe tenerse en cuenta el horizonte de la Revelación y que la catequesis y la evangelización misma deben estar más radicalmente referidas a la Revelación. A eso impulsa la cultura actual, siempre que se la considere sin unilateralidad. Si la primera preocupación es la evangelización (y la ubicación de la catequesis en el proceso de evangelización) - en el fondo una preocupación eclesial - la cultura permanece inexorablemente como punto de llegada; se permanece en la unilateralidad. En efecto, la opción inicial del primado de la evangelización, produce la sensación de que el DC se encuentre ya desde la salida con retraso sobre la cultura. También es cierto que la confrontación con la cultura lanza en todo caso, mensajes de vuelta atrás que inducen a romper con la unilateralidad y a reconsiderar la opción de salida. No en el sentido

10 EG 163.

11 EG 164-165. 
de invertir el esquema, es decir: primero la cultura. Pero, ciertamente, en el sentido de una vuelta al primado de la Revelación.

Pero eso debe ser verificado indagando sobre el primer capítulo del DC, el que da horizonte y contexto a la catequesis. En él se afirma el primado de la evangelización y se da una triangulación entre evangelización, revelación y cultura.

\section{De la evangelización a la (trasmisión de la) Revelación y a la cultura: unilateralidad y apertura}

La preocupación por dar contexto a la catequesis (contexto eclesial, de evangelización, de Iglesia misionera), que se nota presente en el primer capítulo del DC, recoge, como ya hemos señalado, una insistencia que se manifiesta y se profundiza cada vez más en el postconcilio. La renovación de la catequesis, que se había concentrado inicialmente sobre la catequesis como tal (sobre los métodos, sobre los objetivos, sobre los contenidos), se ha abierto gradualmente a las condiciones pastorales y eclesiales. La catequesis profundizaba su identidad cuanto más respiraba aire de conversión pastoral, de Iglesia misionera, abierta, en salida. En efecto, la cualidad de la comunidad cristiana y de su evangelización hace efectiva la calidad de la catequesis. El DC recoge esta sensibilidad e identifica el contexto de la catequesis en la "misión evangelizadora de la Iglesia". Eso viene expresado en el título de la primera parte del DC (que retoma literalmente el título de la primera parte del DGC): "La catequesis en la misión evangelizadora de la Iglesia”.

El capítulo I tiene por objetivo describir tal contexto, abriendo así el camino a la comprensión de la catequesis (es decir a "la identidad de la catequesis", que es el tema del capítulo II). En efecto: siguiendo el contexto del que se parte se perfila luego una específica comprensión de la catequesis. Pero, ¿de qué manera el primer capítulo interpreta el contexto de la catequesis? En realidad, se produce un desplazamiento o una especie de nueva comprensión del título de la primera parte. El título del capítulo I es: "La Revelación y su transmisión". Las palabras clave son pues Revelación y transmisión. Se produce, en realidad, un desplazamiento de la misión evangeli- 
zadora a la Revelación o, más exactamente, a la transmisión de la Revelación. La misión evangelizara viene especificada (e interpretada), como para dar un contexto más específico a la catequesis, en la transmisión de la Revelación (también en esto el DC se halla en plena sintonía con el DGC).

La estructura del capítulo da enseguida a entender que son tres las palabras clave: Revelación, evangelización y cultura, vinculadas entre sí por la idea de la transmisión. El orden de las palabras expresa el movimiento de la reflexión: de la Revelación - que tiene su centro en Cristo y exige una respuesta de fe - a la transmisión de la Revelación - por medio de la evangelización, misión fundamental de la Iglesia - a la evangelización en el mundo contemporáneo - es decir a la confrontación con los desafíos culturales actuales, que abre una nueva etapa evangelizadora. La primera palabra es Revelación, no evangelización (o misión evangelizadora). Pero lo que es primero cronológicamente es, en realidad, segundo en el orden del sentido. El horizonte de sentido, en la reflexión, viene dado por la evangelización, no por la Revelación; viene dado por la evangelización pensada como transmisión de la Revelación. En otras palabras: la Revelación es pensada inmediatamente en la perspectiva de la evangelización y la problemática de la evangelización se convierte en "transmisión de la Revelación” (v. el tercer título en el interior del capítulo).

Este punto es decisivo porque implica una disminución del sentido de la Revelación (que tiene un peso en la aproximación a la cultura). La Revelación, con su "economía” - es "iniciativa del amor de Dios" "orientada a la comunicación, que alcanza su plenitud en Cristo, que se actúa con "acontecimientos y palabras íntimamente ligadas" (12, que pone en valor DV2) - no logra, en el DC, informar de por sí la evangelización; no logra dar forma a la transmisión o a provocar su comprensión. La misma se convierte, en el fondo, en el contenido de la evangelización. Es la preocupación por la evangelización la que se lleva la mejor parte y produce una sutil reducción de la Revelación.

Ello sucede, también, por la presencia de una cierta oscilación, en la comprensión de la Revelación. A veces, el acento sobre la verdad y sobre su conocimiento (por ej.: Dios "ha querido revelar el misterio de 
su voluntad comunicándose él mismo a los hombres" -11); otras veces sobre el amor y sobre la invitación a la comunión ("La Revelación es iniciativa del amor de Dios y está orientada a la comunión” -12). Eso tiene implicaciones también sobre la comprensión del anuncio. En el n. 14 se dice: "El anuncio cristiano comunica el diseño divino"; pero tal diseño viene justamente connotado como un "misterio de amor", por el cual "los hombres, amados por Dios, son llamados a responderle, llegando a ser signo de amor para los hermanos" (14). En realidad, prevalece, tanto en relación con la Revelación como sobre el anuncio, un cierto primado del conocimiento (ciertamente, conocimiento del amor) sobre la iniciativa de amor. Pero es legítimo cuestionarse: ¿si la Revelación es iniciativa de amor, no debería ella alterar y dar sentido al conocimiento mismo? Y también, ¿se anuncia el amor o se ama anunciando? ¿Se responde a Dios ante todo tomando conciencia de su diseño de amor o se responde amando (y por eso se toma conciencia)?

La oscilación ya señalada la reencontramos también en la reflexión sobre "La fe en Jesucristo: la respuesta a Dios que se revela" (es el título de la sección 2). La respuesta de fe de la persona está pensada a partir "de la inquietud que habita en su corazón", pero tal inquietud se ve rápidamente referida a una cuestión, la de la "búsqueda sincera del sentido de la propia existencia (17), que es, en el fondo, cuestión de conocimiento o comprensión. Ello conduce inevitablemente a pensar la Revelación como ofrecimiento de sentido para la vida del hombre, Pero la Revelación, en el fondo, ¿es oferta de sentido? 0 ¿es posibilidad de sentirse amado y riesgo de dejarse amar y de amar?

La fe está pensada en las dos dimensiones, ubicadas en íntima relación entre sí, de la fides qua y de la fides quae (18). Pero, para salir del primado de la comprensión, no es suficiente con subrayar la conexión entre las dos dimensiones, como si fuesen dimensiones iguales. Permanece siempre una diferencia entre el acto de fe y los contenidos de la fe. Por otra parte, la conexión de las dos dimensiones es ya una operación de comprensión: el intelecto (o incluso sólo la intuición) colma la diferencia, que existencialmente permanece.

Pero, en el fondo, el primado de la comprensión había ya sido decidido pensando la Revelación en perspectiva de trasmisión, o sea, 
como contenido de la misión evangelizadora de la Iglesia. ¿No era necesario pensar la evangelización a la luz de la Revelación, es decir, según la Revelación? Sin este horizonte más grande la evangelización permanece con un sutil sentido de unilateralidad. Sigue siendo acción de la Iglesia hacia la cultura. Ciertamente, es acción de la Iglesia, pero de una Iglesia que es mediadora (y busca interceptar) de la acción de Dios; de una Iglesia, también, que mantiene viva la memoria de que Dios habla también desde la cultura.

Pero, cabe subrayar que hay, en todo el capítulo, expresiones que rompen, e incluso fuertemente, la lógica de fondo, y por tanto la unilateralidad. Por ejemplo, las expresiones (dentro de la sección 3 sobre la "transmisión de la Revelación en la fe de la Iglesia") que se abren a la acción del Espíritu: "El Espíritu Santo, verdadero protagonista de toda la misión eclesial, actúa tanto en la Iglesia como en aquellos a los que ella acoge ya que Dios obra en el corazón de cada persona" (23); el mismo Espíritu "despierta el deseo de obrar bien" en el corazón de las personas (23); la Iglesia misma "se enriquece" "en el encuentro con aquellos a los que entrega el Evangelio" (23). El hecho de que tales afirmaciones se encuentren dentro de un planteamiento que se refiere a la trasmisión de la Revelación disminuye un poco su fuerza. La Revelación, que, como se ha dicho, aquí figura sobre todo como contenido, debería, en cambio, (re)significar el sentido mismo del transmitir. Cierto, la Iglesia es, "dócil a las indicaciones del Espíritu Santo, escuchando la Revelación (28), pero, ante todo, ella se ve desafiada a encontrarse tras sus huellas o a convertirse en su huella, y su evangelización debería, en cierto modo, situarse ya sobre las huellas de la obra de Dios en la historia y en las huellas del paso de Dios en la existencia.

La descripción del proceso de evangelización (31ss.), que evoca la fenomenología de la evangelización propuesta por Evangelii Nuntiandi (de la cual se cita el n. 21), tiene sin embargo diversos elementos de ruptura de la unilateralidad. El testimonio implica diálogo, reciprocidad, "disponibilidad para reconocer los signos del bien y de la presencia de Dios en las personas con las que nos encontramos. En efecto, Dios sale al encuentro del corazón mismo de las personas a quienes se quiere comunicar el Evangelio: Él llega siempre primero". 
Esto está vinculado con el primado de la gracia, otro modo de romper la unilateralidad: "El reconocimiento del primado de la gracia es fundamental en la evangelización, desde el primer momento". Se nos ocurre pensar también en los discípulos caminando con todos. "Por lo tanto, los discípulos de Jesús, compartiendo la vida con todos, dan testimonio incluso sin palabras de la alegría del Evangelio que siempre plantea preguntas. El testimonio, que también se expresa como un diálogo respetuoso, se hace anuncio en el momento oportuno" (33). Posteriormente, se podría aquí poner de relieve el sentido del camino con todos (según GS 1) y del diálogo de reciprocidad. En cambio, el recorrido - que va desde la "acción misionera" (que comprende: "testimonio", "sensibilización hacia la fe y hacia la conversión inicial", "tiempo de búsqueda y de maduración") a la "acción catequístico-iniciática” y a la "acción pastoral" (33-35) - parece, no obstante, marcado por la preocupación (eclesial, de evangelización) por que se realice todo el ministerio de la Palabra (36), en sus diversas formas (37).

Así se llega al mundo contemporáneo y a la cultura (la sección 4: "La evangelización en el mundo contemporáneo”). La visión viene dada por la evangelización, incluso la referencia al mundo contemporáneo está como inserto dentro de "una nueva etapa evangelizadora" (38). Pero, también aquí, se ponen en evidencia algunos elementos de ruptura del movimiento unilateral. El tiempo actual, complejo y cambiante, señala el mismo "camino eclesial" que está "caracterizado por dificultades y exigencias de renovación espiritual, moral, pastoral" (38). Aparece de nuevo el reconocimiento del primado del Espíritu, que ofrece una perspectiva de una renovada comprensión de la expresión "nueva evangelización"; la novedad está vinculada a la "acción innovadora del Espíritu del Resucitado" (39). La expresión nueva evangelización se ve en parte liberada de aquel carácter de unilateralidad con respecto a la cultura, que con frecuencia caracterizó el debate de los años pasados; en realidad, hasta pierde fuerza y va como a desembocar en las expresiones, ya consagradas, como "conversión pastoral" o Iglesia "en salida". "La espiritualidad de la nueva evangelización se realiza hoy por una conversión pastoral, mediante la cual la Iglesia es invitada a realizarse en salida, siguiendo un dinamismo que atraviesa toda la Revelación y situándose en un estado 
permanente de misión" (40). Conforme con la perspectiva de la evangelización, los referentes se adjuntan en relación a la situación en la que se encuentran con respecto a la experiencia de fe y eclesial (41).

La orientación sutilmente unilateral se vuelve a encontrar también en la sección sobre "Evangelización de las culturas e inculturación de la fe" (42-47), a pesar de que, de vez en cuando, aparecen importantes elementos de ruptura; por ejemplo, en el número 43, donde se toman distancia de una evangelización entendida como ocupación de territorio. Puesto que la categoría privilegiada de enfoque actual es la de la distancia entre fe y cultura (44), ello lleva a pensar la inculturación de la fe desde la óptica del cuidado de los lenguajes (44), de la atención a la peculiaridad de las situaciones culturales (45), del medirse por los nuevos horizontes de significado (46). En realidad, se advierte una actitud un poco instrumental, que, en el fondo, permanece un tanto externo a la cultura. Se habría podido intensificar el acento sobre el encuentro, sobre dinámicas de reciprocidad, sobre el reconocimiento del protagonismo de las personas, ya que la inculturación se hace desde el interior mismo de las culturas y a partir de los gérmenes de bien y de verdadera humanidad que se abren al descubrimiento de nuevas potencialidades del Evangelio. No obstante, se señala que "Vivimos en la práctica una verdadera revolución antropológica, que trae consecuencias para la experiencia religiosa y que desafía fuertemente a la comunidad eclesial" (46). La cual está vinculada a las profundas transformaciones culturales vinculadas a los "medios de comunicación de masas"; "que han replanteado las coordenadas básicas humanas, yendo más allá de las finalidades propias de la comunicación" (47).

Se advierte una cierta disonancia entre la puesta en evidencia de la radicalidad de los cambios y la vuelta a proponer, en el fondo, el paradigma habitual de evangelización y de catequesis. Ésta debe asumir, ciertamente, algunos "acentos" propios (48), que, en el fondo, nos reconducen al hecho de dejarnos permear por el espíritu misionero. Ello está "al servicio de la nueva evangelización" (48) y está también, como la Iglesia, "en salida misionera" (49). Algunas expresiones hacen pensar que, si se sale de verdad, se rompe la unilateralidad porque se cae en una especie de contra movimiento. Por 
el hecho de estar en salida, la catequesis "está dispuesta escuchar las llamadas de verdad que ya están presentes en las diferentes actividades humanas, con la certeza de que Dios actúa misteriosamente en el corazón de la persona incluso antes que el Evangelio" (50). Es uno de aquellos textos que no sólo atenúan la unilateralidad, sino que ofrecen también apoyo para revisitar el paradigma de la catequesis y de la evangelización.

Un acento importante de la catequesis - es evidente en esto el influjo del magisterio del Papa Francisco - deberá ser la misericordia. Hay algunos apuntes importantes para una "catequesis bajo el signo de la misericordia" (51-52): la misericordia es "centro de la Revelación de Jesucristo"; "la práctica de la misericordia es ya una auténtica catequesis, es catequesis en acto, es el testimonio claro para creyentes y no creyentes"; la nueva evangelización debe usar "el lenguaje de la misericordia" (51). Pero, tales afirmaciones, (sobre todo la primera) deberían ser tomadas en su radicalidad y deberían inspirar el sentido mismo de la catequesis (y también de la Revelación). El hecho mismo de que la misericordia sea un acento de la catequesis y que esté ubicado al final del capítulo es indicativo de su no centralidad. Y sin embargo se abre el camino para repensar radicalmente la catequesis y la evangelización. Lo mismo se puede decir de la "catequesis como "laboratorio" de diálogo" (53-54). Algunas aperturas son importantes, por ejemplo: la invitación a que la catequesis "acentúe" un "estilo dialógico" y la afirmación de que ella es "auténtico "laboratorio" de diálogo", aun cuando se apresure a recomendar "un diálogo pastoral sin relativismos" (54).

El esquema de acercamiento a la cultura, que aparece en el primer capítulo, se vuelve a encontrar coherentemente en todo el DC. Se lo encuentra en el capítulo II que explicita la identidad de la catequesis poniendo el acento en su íntima relación con el kerigma y con su ubicación en el interior del proceso de evangelización. En ese capítulo la referencia a la Palabra de Dios está, en cierto modo, subordinado a la Iglesia evangelizadora, así como en el primer capítulo la Revelación está, en cierto modo, subordinada a la evangelización. El mismo esquema se lo encuentra en tantos puntos neurálgicos del DC: en la comprensión del proceso catequístico (en la segunda parte), allí donde las personas y la experiencia 
se reencuentran, a menudo sutilmente, destinatarias de la preocupación de la trasmisión de la fe; al confrontarnos con los escenarios culturales contemporáneos (capítulo X), allí donde se trata, en el fondo, de alcanzar la cultura; cuando se describe la inculturación de la fe (el tema está en el capítulo XI pero circula por todo el texto). Préstese atención: el sentido de la unilateralidad no se halla en el plano metodológico y mucho menos en el plano de la pastoral catequística, que intentan articularse en el sentido de la reciprocidad y del caminar juntos, sino en la lógica de fondo, justo en el horizonte. Se podrían indicar, además, tantos otros puntos de ruptura de la lógica de fondo, diseminados en todo el documento, así como los hemos encontrado en el capítulo I. Es necesario también señalar ulteriormente que el problema no está solamente en el DC sino que está, ante todo, en la tradición catequística posconciliar que se ha asentado en una dirección que el DC no hace más que recoger y relanzar.

\section{La clave cultural y el tema teológico}

Ahora, debemos preguntarnos: ¿Cómo superar la sutil unilateralidad que hemos encontrado? ¿Cómo volver a comprender la relación entre evangelización y cultura? ¿Cómo hacerlo sin aplastar la evangelización bajo la cultura y evitando que los criterios de la evangelización y de la catequesis sean dictados por la cultura? ?2 $^{12}$ asimetría, o una diferencia, entre evangelización y cultura debe ser mantenida. En catequesis, es el Evangelio quien inspira los procesos educativos y comunicativos ${ }^{13}$. Además, la evangelización será siempre propositiva; la catequesis no deberá nunca renunciar a proponer el Evangelio. Pero, es posible ser propositivo dentro de un horizonte relacional de verdadera reciprocidad, mientras se habita el terreno común de nuestra común humanidad.

A este terreno deberá ser reconducida la diferencia evangelización-cultura y todas las diferencias relacionadas con ella (fe-cultura, creyentes-no creyentes, Iglesia-sociedad), en caso contrario todas

12 R. Fisichella manifiesta, justamente, esta preocupación en Fisichella, Guida alla lettura, 13-14.

13 Y que preside también a la valoración de las contribuciones de las ciencias humanas, como explica justamente DC 147. 
ellas se convierten en dualismos. Deberán ser reconducidas a la diferencia inscrita en el terreno de la común humanidad. Ese terreno, de hecho, no es neutro. Se ve, todo él, impregnado por las huellas de la obra de Dios (de la creación y de la redención). Cada uno de nosotros se halla posicionado sobre dicho terreno: o de la parte del bien o del mal, de la vida o de la muerte; está ya habitando las huellas de la obra de Dios o está huyendo de ellas. La vida está impregnada siempre de estos dilemas o de esta diferencia. El Evangelio deberá aportar luz de discernimiento. Pero es como si la iluminación viniese, al mismo tiempo, de fuera y de dentro, de lo alto y de abajo. El Evangelio ilumina sobre la diferencia, mientras se habita la diferencia; habitar la diferencia es permitir al evangelio que resuene.

En efecto, la Evangelii Gaudium crea una profunda conexión entre la evangelización y los dilemas de nuestra humanidad, evocados tanto a nivel personal como a nivel cultural (trasversales para creyentes y no creyentes): ¿la cultura del encuentro o del descarte? ¿del Dios dinero o del compartir? La Laudato si y la Fratelli tutti, de igual modo, nos reconducen a los desafíos comunes: aquellos que provienen del habitar la casa común y de aquellos relativos a la construcción de un mundo fraterno. Son desafíos que no pueden ser dejados de lado como consecuentes con respecto a la acogida del Evangelio o como la dimensión social de la experiencia de fe, como insinúa el DC (que, en esto, no hace suya a fondo la provocación del Papa Francisco), sino como el horizonte (de verdad de lo humano y de restitución a la obra de Dios, al mismo tiempo) de la resonancia del Evangelio y de todo crecimiento en la fe.

El progresivo camino de contextualización de la catequesis en el postconcilio, anteriormente citado, espera un paso al frente. Anteriormente aprendimos a pensar la catequesis en el contexto eclesial; luego, conforme la instancia misionera urgía, aprendimos a pensar la catequesis en el proceso de evangelización, es decir en una Iglesia misionera y en salida. Pero, hasta aquí, el horizonte sigue siendo el nuestro; y la unilateralidad es inevitable. El paso siguiente es pensar la catequesis (y la evangelización) sobre el terreno de la común humanidad $^{14}$. Pero no ingenuamente. El acercamiento a la humanidad 14 Referencia a S. Currò, Giovani, Chiesa e comune umanità. Percorsi di teologia pratica sulla 
deberá ser en la fe, a la luz de la Palabra, en el primado de la Revelación. Para que eso sea posible, la evangelización (y por tanto también la catequesis) deberá interceptar el movimiento de la Revelación. En esto, la catequesis, por relación constitutiva con la Palabra, desempeña un papel fundamental. Ella que es, por definición, resonancia de la Palabra, ayuda a liberar el primado y el sentido de la Revelación; al mismo tiempo vive de ello.

Aquí se halla la clave teológica del DC, pero también de la pastoral catequística y de todo el proceso de renovación de la catequesis. El DC comienza por la Revelación, pero sin comenzar de verdad desde la Revelación, porque de hecho comienza por la misión evangelizadora de la Iglesia. Pero la misión evangelizadora vive de la Revelación. Y la Revelación es irreducible a contenido a ser transmitido, aun cuando se haga contenido. La evangelización vive siempre de una iniciativa de amor de Dios que no se deja capturar, que se ha manifestado en Cristo y está codificada en las Escrituras, pero que atraviesa también la historia (incluida la actual). La cultura (¡de nuevo, incluida la actual!) no puede ser elemento extrínseco a la Revelación. Cristo, que también juzga, da sentido y constituye una piedra de tropiezo para cada cultura; nos alcanza también desde el corazón de la cultura.

Volver a encontrar el primado de la Revelación significa también reencontrar la estructura de revelación de la existencia, si así se puede decir; o sea reencontrar aquella iniciativa diversa (llamada de amor, invitación, promesa, provocación o vocación) que vive en nosotros radicalmente, donde la fuga de Dios y la fuga de nosotros mismos y de los otros se cruzan misteriosamente y donde un reclamo pascual (de paso de la muerte a la vida) está ya inscrito y tiene también necesidad de ser despertado y revelado. La catequesis tiene la tarea de hacer resonar la Pascua mientras se despierta el dinamismo pascual de la existencia. En este sentido, una apertura interesante (para ser acogida, trabajada, profundizada) se encuentra en la conclusión del DC. "La catequesis, eco de la Pascua en el corazón de la persona, invita incesantemente a salir de sí mismo para encontrarse con el Viviente, Aquel que da la vida en plenitud" (426).

conversione pastorale, Elledici, Torino 2021, 151-179 (el capítulo sobre "Recomprender el sentido de la catequesis: a partir de la provocación del Papa Francisco”). 